\title{
3D Printed Calibration Micro-phantoms for Validation of Super-Resolution Ultrasound Imaging
}

Ommen, Martin Lind; Schou, Mikkel; Beers, Christopher; Jensen, Jørgen Arendt; Larsen, Niels Bent; Thomsen, Erik Vilain

\section{Published in:}

Proceedings of the IEEE International Ultrasonic Symposium 2019

Link to article, DOI:

10.1109/ULTSYM.2019.8926292

Publication date:

2019

Document Version

Peer reviewed version

Link back to DTU Orbit

Citation (APA):

Ommen, M. L., Schou, M., Beers, C., Jensen, J. A., Larsen, N. B., \& Thomsen, E. V. (2019). 3D Printed Calibration Micro-phantoms for Validation of Super-Resolution Ultrasound Imaging. In Proceedings of the IEEE International Ultrasonic Symposium 2019 (pp. 1212-1215). IEEE.

https://doi.org/10.1109/ULTSYM.2019.8926292

\section{General rights}

Copyright and moral rights for the publications made accessible in the public portal are retained by the authors and/or other copyright owners and it is a condition of accessing publications that users recognise and abide by the legal requirements associated with these rights.

- Users may download and print one copy of any publication from the public portal for the purpose of private study or research.

- You may not further distribute the material or use it for any profit-making activity or commercial gain

- You may freely distribute the URL identifying the publication in the public portal 


\title{
3D Printed Calibration Micro-phantoms for Validation of Super-Resolution Ultrasound Imaging
}

\author{
$\underline{\text { Martin Lind Ommen }}^{1}$, Mikkel Schou ${ }^{1}$, Christopher Beers ${ }^{2}$, Jørgen Arendt Jensen ${ }^{1}$, Niels Bent Larsen ${ }^{1}$, and \\ Erik Vilain Thomsen ${ }^{1}$ \\ ${ }^{1}$ Department of Health Technology, Technical University of Denmark, Kgs. Lyngby, Denmark \\ ${ }^{2}$ BK Medical, State College, Pennsylvania, USA
}

\begin{abstract}
This study evaluates the use of 3D printed phantoms for super-resolution ultrasound imaging (SRI) algorithm calibration. Stereolithography is used for printing calibration phantoms containing eight randomly placed scatterers of nominal size $205 \mu \mathrm{m} \times 205 \mu \mathrm{m} \times 200 \mu \mathrm{m}$. The purpose is to provide a stable reference for validating new ultrasonic imaging techniques such as SRI. SRI algorithm calibration is demonstrated by imaging a phantom using a $\lambda / 2$ pitch $3 \mathrm{MHz} 62+62$ row-column addressed (RCA) ultrasound probe. As the imaging wavelength is larger than the dimensions of the scatterers, they will appear as single point spread functions in the generated volumes. The scatterers are placed with a minimum separation of $3 \mathrm{~mm}$ to avoid overlap of the point spread functions of the scatterers. 640 volumes containing the phantom features are generated, with an intervolume uniaxial movement of $12.5 \mu \mathrm{m}$, emulating a flow velocity of $2 \mathrm{~mm} / \mathrm{s}$ at a volume frequency of $160 \mathrm{~Hz}$. A superresolution pipeline is applied to the obtained volumes to localise the positions of the scatterers and track them across the 640 volumes. The standard deviation of the variation in the scatterer positions along each track is used as an estimate of the precision of the super-resolution algorithm, and was found to be between the two limiting estimates of $(x, y, z)=(17.7,27.6,9.5) \mu \mathrm{m}$ and $(x, y, z)=(17.3,19.3,8.7) \mu \mathrm{m}$. In conclusion, this study demonstrates the use of $3 \mathrm{D}$ printed phantoms for determining the precision of super-resolution algorithms.
\end{abstract}

Index Terms-3D printing, stereolithography, phantom, hydrogel, calibration, resolution, ultrasound

\section{INTRODUCTION}

Super-resolution ultrasound imaging (SRI) has emerged over the last few years as a non-invasive method for imaging of the smallest sections of the vasculature [1], [2], [3]. This is enabled by the use of micrometer sized bubbles acting as a contrast agent and is believed to be capable of passing vessels as small as $10 \mu \mathrm{m}$. Tracking of individual contrast bubbles over a period of time can reveal images of the vasculature. Structures well below the diffraction limit of conventional ultrasound have been shown. However, a fundamental problem is to validate the spacial accuracy of these new techniques. Biological structures are often very complex in geometry with liquid flow and tissue motion as added complications. This means that the ground truth of the imaging system accuracy can never be obtained on biological samples. Instead, it is attained using phantoms designed for ultrasound. With the introduction of SRI, the requirements for phantoms focus on the scale of features, to utilize the increased resolution, while still being able to replicate the dimensionality of vascular networks. Since the introduction of SRI development, the algorithm principles have been demonstrated in fairly few phantom studies. The phantoms have all contained channels with varying dimensions, fabricated using different methods. In one case, the channel dimensions were decreased to $40 \times 80 \mu \mathrm{m}^{2}$ [4] by utilizing the high resolution of silicon micro-fabrication methods. In practice, this enforces a limit of the channels at most being in a few planes, and true 3D replication of structures will be very limited. Other examples of phantoms consisting of tubes have been presented by Viessmann et al. (3 $\mathrm{mm}$ diameter) [5] and by Christensen-Jeffries et al. (200 $\mu \mathrm{m}$ diameter) [6]. These structures are larger than the vessels of interest, which would be smaller than $100 \mu \mathrm{m}$ in diameter. One of the most recent superresolution phantom studies was made using a 2D sparse array transducer imaging two cellulose tubes arranged in a double helix pattern to create a three-dimensional phantom structure [7]. A common element of the mentioned phantom studies is that they are channel based and meant to provide a known outer limit for the localization of the tracked microbubbles. However, that does not allow for exact control of the microbubbles' positions within the channels. Therefore the spatial position of the imaging source is still not fully known.

3D printing is a promising new method for creating ultrasound phantoms. It allows for the flexibility of fabricating complex 3dimensional structures, as well as printing of very small features in the sub- $100 \mu \mathrm{m}$ range [8]. Recently, the first 3D printed phantoms for ultrasound were demonstrated [9], albeit seemingly not with SRI vascular replication in mind. The phantoms contained highly scattering solid features as small as $30 \times 50 \mu \mathrm{m}^{2}$ in cross section, demonstrating the exciting potential for point spread function evaluation provided by the method. We recently demonstrated 3D printing by stereolithography to obtain small cavities and channels in a hydrogel, which is suitable for SRI ultrasound [10]. It was shown that small cavities in the 3D printed hydrogel will scatter sound, and therefore be visible in ultrasound images. Thus, in a similar manner to solid encapsulation 3D printing by Jacquet et al. [9], it is possible 


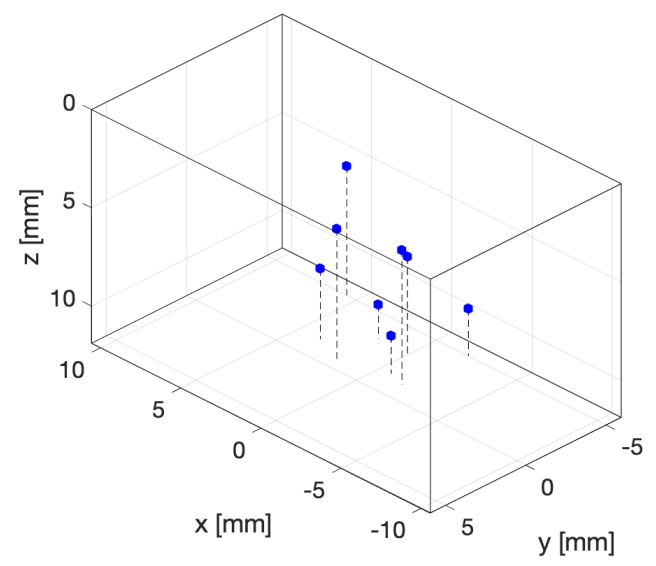

Figure 1. The designed layout of the scatterers within the $\sim 21.1 \times 11.9 \times 11.9 \mathrm{~mm}^{3}$ phantom. The drop-lines are included to aid 3D perception, and end on the $z=10 \mathrm{~mm}$ plane.

to fixate small scatterers within the hydrogel. These structures will be stable in time, and can be imaged repeatedly, in direct contrast to microbubbles in small channels. In this paper, we demonstrate through 3D SRI how these stable structures can be used to determine the precision of the SRI hardware and algorithms. This method can potentially be used to demonstrate local variations in the precision of the algorithms based on the scatterer's position within the localization field of view.

\section{MATERiALS AND MethodS}

\section{A. Fabrication of the phantoms}

Calibration phantoms were fabricated by stereolithographic 3D printing of an aqueous solution of poly(ethylene glycol) diacrylate (PEGDA, $\mathrm{M}_{\mathrm{n}} 700 \mathrm{~g} / \mathrm{mol}$, 455008, Sigma-Aldrich) to form a hydrogel solid. Stereolithography is based on printing a stack of individual thin layers of materials, calling for prior digital slicing of the targeted 3D design into separate layer designs matching the printing system. The method and components have previously been presented in more detail [10]. To determine the precision of the SRI algorithms, a phantom containing eight randomly placed scatterers was printed. The outer dimensions of the phantom was $21.1 \times 11.9 \times 11.9 \mathrm{~mm}^{3}$ with each scatterer being $205 \times 205 \times 200 \mu \mathrm{m}^{3}$. While the printing setup allows for printing significantly smaller scatterers, it is necessary with an increased size in order to obtain a reflection with intensity larger than that of bulk noise scatterers in the phantom. The scatterers will appear as single point spread functions in regular B-mode volumes when the imaging wavelength is larger than the scatterer size, in this case for any frequency below $\approx 6 \mathrm{MHz}$. They were placed with a minimum separation distance of $3 \mathrm{~mm}$ which will eliminate overlapping signals for any frequency above $0.5 \mathrm{MHz}$. The designed layout is shown in Fig. 1.

\section{B. Experimental setup and procedure}

A custom built stage was designed to mount the $3 \mathrm{D}$ printed phantom. A 3D printed frame fitted to the phantom was used to mount it on top of an absorbing polyurethane rubber sheet (Sorbothane, Inc., Kent, Ohio, USA) on the stage, which can then be submerged in water. Subsequently, the stage was mounted on a 8 MR190-2-28 rotation stage $\left(0.01^{\circ}\right.$ resolution $)$ combined with a 8MTF-75LS05 $x-y$ translation stage $(0.31 \mu \mathrm{m}$ resolution) (Standa, Vilnius, Lithuania), which in turn was mounted on a Newport PG Series floating optical table (Irvine, California) for stability.

Two separate experiments were conducted. The translation stage was used to move the phantom relative to the ultrasound probe along a single axis; in the first experiment along the $x$ axis, and in the second experiment along the $y$-axis. In both experiments, the inter-volume stage movement was $12.5 \mu \mathrm{m}$, which emulates a $2 \mathrm{~mm} / \mathrm{s}$ velocity at a $160 \mathrm{~Hz}$ volume rate. The imaging probe was a prototype $62+62$ elements 3 $\mathrm{MHz}$ piezo-electric, row-column addressed (RCA) transducer [11]. The probe was connected to the experimental scanner SARUS [12], a system capable of storing all channel data to be processed offline. A single frame consisted of 32 defocused emissions using a synthetic aperture (SA) imaging approach. Rows were transmitting and columns receiving, resulting in 62 channels in receive per emission. The data was passed to the SRI pipeline, described in Section II-C. The phantom was stationary when a frame was measured to avoid intra-frame motion artefacts. In total 640 volumetric frames were acquired over 640 positions. The volumetric frames were then passed to the SRI pipeline [13].

\section{Super-resolution Pipeline}

The super resolution pipeline has three steps. The first step is the SA beamforming. A single frame consists of a volume with dimensions of $14.86 \times 14.86 \times 7.43 \mathrm{~mm}^{3}$, corresponding to $61 \times 61 \times 243$ voxels. The volume is created by beamforming all 32 emissions in a frame with a specialized beamformer [14] implemented on a GPU [15]. The 32 beamformed volumes were then summed to reveal a single high resolution volume. The volume was dynamically focused in receive (F-number of 1.5) and synthetically in transmit (F-number of 1), with an optimized sequence for SA B-mode. All 640 frames were beamformed and a stationary echo filter was applied to remove stationary tissue. However since the entire phantom is moving in this experiment, this step had no effect on the results. The third step is to locate the point scatterers from local maxima. The peak location is interpolated using a second order polynomial in all three dimensions to attain sub-pixel positioning. The output from this stage is a series of 3-D coordinates $\left\{x_{p}, y_{p}, z_{p}\right\}$ for all detected points. The coordinates can then be collected across all imaged frames to form tracks. The pipeline was implemented in MATLAB, and processing was performed offline [13]. 


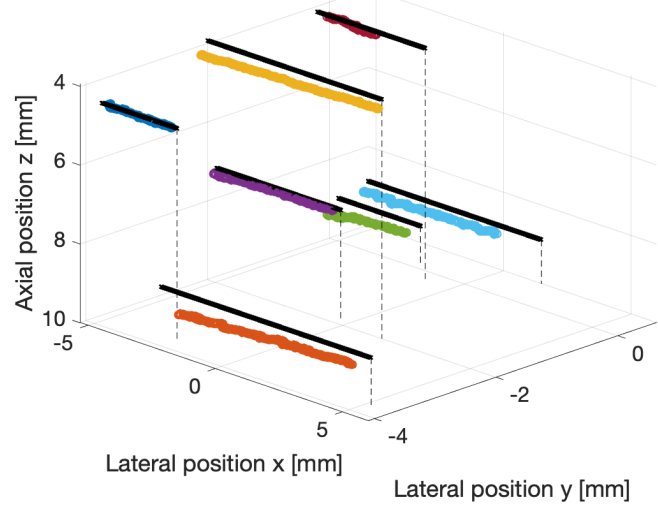

(a) Motion along the $x$-axis

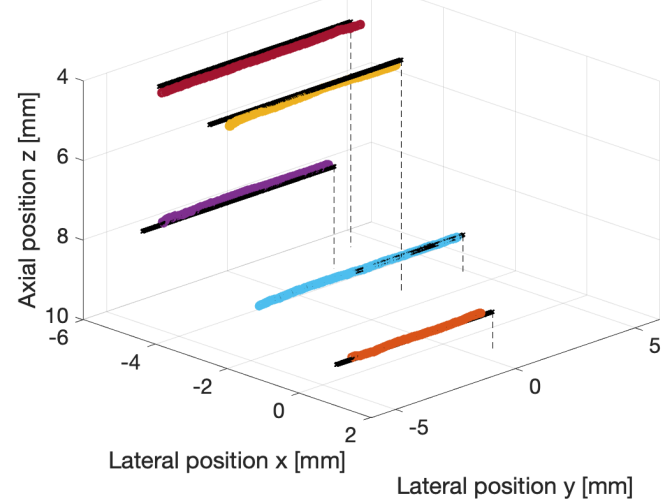

(b) Motion along the $y$-axis

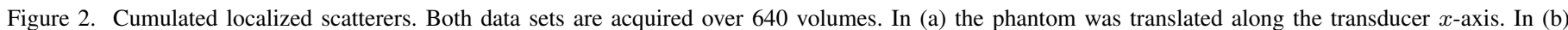

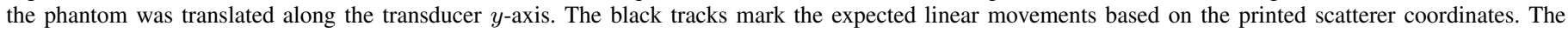
drop-lines are included to aid 3D perception and end on the $z=10 \mathrm{~mm}$ plane.

\section{RESULTS}

\section{A. Scatterer localisation}

Fig. 2 shows the cumulated localized positions of the 3D printed scatterers after acquisition of the 640 volumes. The coloured tracks mark the super-localized tracks of scatterers, while the black tracks mark the expected tracks based on the design coordinates. The horizontal field of view of the plots has been limited to the data tracks. The actual horizontal field of view of the probe is $14.86 \times 14.86 \mathrm{~mm}^{2}$. For the movement along the $x$-axis (Fig. 2(a)), seven of the eight printed scatterers have been correctly localized, and for the movement along the $y$-axis (Fig. 2(b)), five of the eight printed scatterers have been correctly localized. While the same phantom was used, differences in the point spread function of the probe based on the position within the imaged volume could mean that slight differences in the starting position of the phantom could result in one experiment correctly detecting more tracks than the other. This can also be the reason for the eighth scatterer not being detected in either experiment. Furthermore, two additional tracks which were not associated with the printed scatterers, have been omitted from the plots and analyses. A possible explanation for these localizations could be print errors, resulting in additional cavities in the phantom which act as extra scatterers. While they would be expected to follow the same trajectories as the designed scatterers, the exact shape of them is unknown. If a printing error is significantly larger than the wavelength itself, localisation of the centroid might be ambiguous. Therefore, the analysis has been limited to the designed scatterers.

\section{B. Precision}

The precision of the SRI algorithm can be determined as the localisation variation around the expected trajectories of the

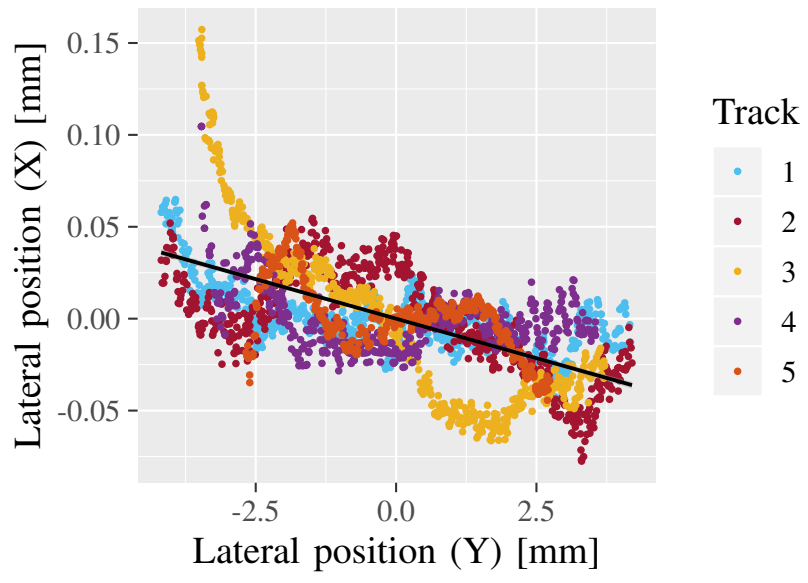

Figure 3. $y-x$ cross-plane for the tracks with motion along the $y$-axis. Note that the axes are not equally scaled, and the misalignment of the track movement to the transducer axes is small.

tracks. While the movement of the tracks should be solely along the $x$ - and $y$-axes respectively, misalignment between the translation stage and the transducer axes could result in a slight offset of the axes. This can be seen in Fig. 3 for the tracks with motion along the $y$-axis, in which the $y-x$ cross-plane is shown.

The colour of the points indicate tracks from different scatterers matched to the corresponding tracks in Fig. 2(b), and the black line is the least squares fit to the localized positions, representing the average trajectory of the points, which in this case is not perfectly along the $y$-axis. It should be noted that the axes are not equally scaled, so the misalignment is small. A logical argument can be made for fitting to the data from all tracks combined, instead of the individual tracks, since the scatterers are fixed and are translated together, which therefore provides a good approximation for the average trajectory. However, close 
Table I

ESTIMATED PRECISION FOR THE SUPER-RESOLUTION ALGORITHM.

\begin{tabular}{lrr}
\hline & $\begin{array}{r}\text { Average } \\
\text { trajectory }\end{array}$ & $\begin{array}{r}\text { Individual } \\
\text { trajectory }\end{array}$ \\
\hline$x[\mu \mathrm{m}]$ & 17.7 & 17.3 \\
$y[\mu \mathrm{m}]$ & 27.6 & 19.3 \\
$z[\mu \mathrm{m}]$ & 9.5 & 8.7 \\
\hline
\end{tabular}

examination of Fig. 3, shows that although the individual tracks roughly follow the black lines, they are not perfectly parallel, but at a small angle to each other, with the tracks crossing the average trajectory at different angles. The same tendency is observed for the $x$-motion tracks. This can indicate distortion in the beamforming.

The standard deviation of the residuals is used as an estimate for the precision of the SRI algorithm. The tracks with movement along the $x$-axis are used to estimate the precision in $y$ and $z$, and the tracks with movement along the $y$-axis are used to estimate the precision in $x$ and $z$. One of the five tracks for the $y$-dataset was omitted due to it not following a normal distribution, for an unknown reason. Since the tracks are not parallel, using the average trajectory would make the deviations from different tracks represent different normal distributions, and therefore not be a single characteristic property of the pipeline. Table I shows two sets of the estimated precision of the SRI pipeline averaged across all tracks: one set uses the average trajectory to determine the deviations; the other uses the individual trajectories.

\section{Discussion AND CONCLUSION}

We have presented a 3D printed phantom for SRI algorithm calibration. The phantom contains fixated scatterers measuring $205 \times 205 \times 200 \mu \mathrm{m}^{3}$. By using fixated scatterers instead of micro-bubbles in a phantom-tube, the scatterers will be stable in time, and can therefore be used for calibration of the SRI algorithms. The phantom was imaged using a $\lambda / 2$ pitch $3 \mathrm{MHz} 62+62$ PZT row-column addressed (RCA) probe. The beamformed volumes were processed in an SRI pipeline, tracking the scatterers as they were moved with a translation stage. The localised positions were supposed to be detected along parallel trajectories due to the movement being induced by a translation stage. However, they were found not to be parallel, which likely indicates distortion of the beamforming. Therefore, the correct estimate of the precision is likely in between the estimate using the common trajectory $(x, y, z)=(17.7,27.6,9.5) \mu \mathrm{m}$, and the individual trajectories $(x, y, z)=(17.3,19.3,8.7) \mu \mathrm{m}$. These results demonstrate the novel use of 3D printed phantoms for calibration of SRI algorithms.

\section{ACKNOWLEDGMENT}

This work was financially supported by grant 7050-00004B from Innovation Fund Denmark, and from BK Medical, Herlev,
Denmark.

\section{REFERENCES}

[1] K. Christensen-Jeffries, R. J. Browning, M. X. Tang, C. Dunsby, and R. J. Eckersley, "In vivo acoustic super-resolution and super-resolved velocity mapping using microbubbles," IEEE Trans. Med. Imaging, vol. 34, no. 2, p. $6908009,2015$.

[2] M. A. O'Reilly and K. Hynynen, "A super-resolution ultrasound method for brain vascular mapping," Med. Phys., vol. 40, no. 11, pp. 1-7, 2013.

[3] C. Errico, J. Pierre, S. Pezet, Y. Desailly, Z. Lenkei, O. Couture, and M. Tanter, "Ultrafast ultrasound localization microscopy for deep superresolution vascular imaging," Nature, vol. 527, no. 7579, pp. 499-502, 2015.

[4] Y. Desailly, O. Couture, M. Fink, and M. Tanter, "Sono-activated ultrasound localization microscopy," Appl. Phys. Lett., vol. 103, no. 17, p. 174107, 2013

[5] O. M. Viessmann, R. J. Eckersley, K. Christensen-Jeffries, M. X. Tang, and C. Dunsby, "Acoustic super-resolution with ultrasound and microbubbles," Phys. Med. Biol., vol. 58, no. 18, pp. 6447-6458, 2013.

[6] K. Christensen-Jeffries, J. Brown, P. Aljabar, M. Tang, C. Dunsby, and R. J. Eckersley, "3-D in Vitro Acoustic Super-Resolution and SuperResolved Velocity Mapping Using Microbubbles," IEEE Trans. Ultrason. Ferroelectr. Freq. Control, vol. 64, no. 10, pp. 1478-1486, 2017.

[7] S. Harput, K. Christensen-Jeffries, J. Brown, J. Zhu, G. Zhang, C. H. Leow, M. Toulemonde, A. Ramalli, E. Boni, P. Tortoli, R. J. Eckersley, C. Dunsby, and M. X. Tang, "3-D Super-Resolution Ultrasound Imaging Using a 2-D Sparse Array with High Volumetric Imaging Rate," IEEE Int. Ultrason. Symp. IUS, vol. 2018-Octob, pp. 23-26, 2018.

[8] H. Gong, B. P. Bickham, A. T. Woolley, and G. P. Nordin, "Custom 3D printer and resin for $18 \mu \mathrm{m} \times 20 \mu \mathrm{m}$ microfluidic flow channels," Lab Chip, vol. 17, no. 17, pp. 2899-2909, 2017. [Online]. Available: http://dx.doi.org/10.1039/C7LC00644F

[9] J. R. Jacquet, F. Ossant, F. Levassort, and J. M. Grégoire, "3-D-Printed Phantom Fabricated by Photopolymer Jetting Technology for HighFrequency Ultrasound Imaging," IEEE Trans. Ultrason. Ferroelectr. Freq. Control, vol. 65, no. 6, pp. 1048-1055, 2018.

[10] M. L. Ommen, M. Schou, R. Zhang, C. A. Villagomez Hoyos, J. A. Jensen, N. B. Larsen, and E. V. Thomsen, "3D Printed Flow Phantoms with Fiducial Markers for Super-Resolution Ultrasound Imaging," IEEE Int. Ultrason. Symp. IUS, vol. 2018-Octob, p. 8580217, 2018.

[11] H. Bouzari, M. Engholm, S. I. Nikolov, M. B. Stuart, E. V. Thomsen, and J. A. Jensen, "Imaging Performance for Two Row-Column Arrays," IEEE Trans. Ultrason. Ferroelectr. Freq. Control, vol. 66, no. 7, pp. 1209-1221, 2019.

[12] J. A. Jensen, H. Holten-Lund, R. T. Nilsson, M. Hansen, U. D. Larsen, R. P. Domsten, B. G. Tomov, M. B. Stuart, S. I. Nikolov, M. J. Pihl, Y. Du, J. H. Rasmussen, and M. F. Rasmussen, "Sarus: A synthetic aperture real-time ultrasound system," IEEE Trans. Ultrason. Ferroelectr. Freq. Control, vol. 60, no. 9, pp. 6587394, 1838-1852, 2013.

[13] J. A. Jensen, M. L. Ommen, S. H.Øygard, M. Schou, T. Sams, M. B. Stuart, C. Beers, E. V. Thomsen, N. B. Larsen, and B. G. Tomov, "Threedimensional super resolution imaging using a row-column array," IEEE Trans. Ultrason. Ferroelectr. Freq. Control, p. Accepted for publication, 2019.

[14] M. F. Rasmussen, T. L. Christiansen, E. V. Thomsen, and J. A. Jensen, "3$\mathrm{d}$ imaging using row-column-addressed arrays with integrated apodization. part i: Apodization design and line element beamforming," IEEE Trans. Ultrason. Ferroelectr. Freq. Control, vol. 62, no. 5, pp. 947-958, 2015.

[15] M. B. Stuart, M. Schou, and J. A. Jensen, "Row-column beamforming with dynamic apodizations on a gpu," Proceedings of Spie, vol. 10955, p. 109550Q, 2019. 